\title{
Intraoperative Transfusion of Fresh Frozen Plasma Predicts Morbidity Following Partial Liver Resection for Hepatocellular Carcinoma
}

\author{
Jan Bednarsch ${ }^{1} \cdot$ Zoltan Czigany $^{1} \cdot$ Isabella Lurje ${ }^{1}$ - Christian Trautwein ${ }^{2} \cdot$ Tom Lüdde $^{2}$ - Pavel Strnad ${ }^{2}$. \\ Nadine Therese Gaisa ${ }^{3}$. Alexandra Barabasch ${ }^{4}$. Philipp Bruners ${ }^{4}$. Tom Ulmer ${ }^{1}$. Sven Arke Lang ${ }^{1}$. \\ Ulf Peter Neumann ${ }^{1,5} \cdot$ Georg Lurje ${ }^{1,6}$ (iD
}

Received: 22 January 2020 / Accepted: 13 May 2020 / Published online: 3 June 2020

(C) 2020 The Author(s)

\begin{abstract}
Background The reduction of perioperative morbidity is a main surgical goal in patients undergoing partial hepatectomy for hepatocellular carcinoma (HCC). Here, we investigated clinical determinants of perioperative morbidity in a European cohort of patients undergoing surgical resection for HCC.

Methods A total 136 patients who underwent partial hepatectomy for HCC between 2011 and 2017 at our institution were included in this analysis. The associations between major surgical complications (Clavien-Dindo $\geq 3$ ) and overall morbidity (Clavien-Dindo $\geq 1$ ) with clinical variables were assessed using univariate and multivariable binary logistic regression analysis. Results Multivariable analysis identified the Child-Pugh-Score (CPS, HR = 3.23; $p=0.040)$, operative time (HR= 5.63; $p=$ 0.003 ), and intraoperatively administered fresh frozen plasma (FFP, HR $=5.62 ; p=0.001)$ as independent prognostic markers of major surgical complications, while only FFP $(\mathrm{HR}=6.52 ; p=0.001)$ was associated with morbidity in the multivariable analysis. The transfusion of FFP was not associated with perioperative liver functions tests.

Conclusions The intraoperative administration of FFP is an important independent predictor of perioperative morbidity in patients undergoing partial hepatectomy for HCC.
\end{abstract}

Keywords Hepatocellular carcinoma $(\mathrm{HCC}) \cdot$ Fresh frozen plasma (FFP) $\cdot$ Perioperative morbidity

\begin{tabular}{llll}
\multicolumn{2}{l}{ Abbreviations } & CPS & Child Pugh Score \\
ALT & Alanine aminotransferase & CI & Confidence interval \\
AP & Alkaline phosphatase & CRP & C-reactive protein \\
ASA & American society of anesthesiologists & cmCT & Contrast material enhanced computed \\
AST & Aspartate aminotransferase & & tomography \\
BMI & Body mass index & CUSA & Cavitron Ultrasonic Surgical Aspirator \\
CCI & Comprehensive complication index & CVP & Central venous pressure
\end{tabular}

Electronic supplementary material The online version of this article (https://doi.org/10.1007/s11605-020-04652-0) contains supplementary material, which is available to authorized users.

Georg Lurje

georg.lurje@charite.de; https://www.hopeliver.com

1 Department of Surgery and Transplantation, University Hospital RWTH Aachen, Aachen, Germany

2 Department of Medicine III, University Hospital RWTH Aachen, Aachen, Germany

3 Institute of Pathology, University Hospital RWTH Aachen, Aachen, Germany
4 Department of Radiology, University Hospital RWTH Aachen, Aachen, Germany

5 Department of Surgery, Maastricht University Medical Centre (MUMC), Maastricht, Netherlands

6 Department of Surgery, Campus Charité Mitte | Campus Virchow-Klinikum, Charité-Universitätsmedizin Berlin, Augustenburger Platz 1, 13353 Berlin, Germany 


$\begin{array}{ll}\text { DFS } & \text { Disease-free survival } \\ \text { ECOG } & \text { Eastern Cooperative Oncology Group } \\ \text { FFP } & \text { Fresh frozen plasma } \\ \text { FLR } & \text { Future liver remnant } \\ \text { GCP } & \text { Good clinical practice } \\ \text { GGT } & \text { Gamma glutamyltransferase } \\ \text { HCC } & \text { Hepatocellular carcinoma } \\ \text { HIDA } & \text { Hepato-imino diacetic acid } \\ \text { INR } & \text { International normalized ratio } \\ \text { LiMAx } & \text { Maximum liver function capacity } \\ \text { MELD } & \text { Model of end-stage liver disease } \\ \text { MRI } & \text { Magnetic resonance imaging } \\ \text { OLT } & \text { Orthotopic liver transplantation } \\ \text { OS } & \text { Overall survival } \\ \text { POD } & \text { Postoperative day } \\ \text { TEG } & \text { Thromboelastography } \\ \text { U H - } & \text { University Hospital Rheinisch-Westfälische } \\ \text { RWTH } & \text { Technische Hochschule } \\ \text { UICC } & \text { Union for international cancer control }\end{array}$

\section{Introduction}

Hepatocellular carcinoma (HCC) is the fifth most common malignancy worldwide and its mortality ranks third among all solid tumors, behind only carcinomas of the lung and the colon. ${ }^{1,2}$ Due to the underlying chronic liver disease in most patients with HCC, orthotopic liver transplantation (OLT) is considered the treatment of choice since it treats both underlying liver disease and the malignant tumor. However, liver resections are increasingly utilized, since the availability of liver allografts remains low in many developed countries. ${ }^{3-5}$ Correspondingly, a number of studies demonstrated that partial hepatectomy can be carried out safely even in patients with advanced liver disease. ${ }^{6-8}$ This development is based on an improved preoperative assessment of patient-related risk factors for dismal operative outcome and the introduction of novel surgical techniques. Recent advancements in the modern era of HCC-surgery include several strategies for the dynamic assessment of the liver function and the implementation of advanced laparoscopic liver surgery. ${ }^{9,10}$ While the latter resulted in comparable oncological results with reduced intraoperative blood loss and improved postoperative recovery, dynamic liver function tests such as the LiMAx (maximum liver function capacity), indocyanine green, and the hepatoimino diacetic acid (HIDA) test ${ }^{9,11-13}$ provide a dynamic estimate of the functional liver reserve and improve the preoperative risk evaluation in these patients. ${ }^{14,15}$

Even though partial liver resection for HCC has developed into a safe and reasonable approach in these patients, the ideal management of surgical morbidity remains subject of ongoing debate. ${ }^{16}$ Recent data show the prognostic significance of perioperative complications in terms of oncologic outcomes such as disease-free (DFS) and overall survival (OS) in HCC. ${ }^{17-19}$

Here, we aim to investigate clinical factors associated with perioperative morbidity in a European cohort of patients undergoing surgical resection for HCC.

\section{Patients and Methods}

\section{Patients}

Between 2011 and 2017, one hundred thirty-six $(n=136)$ patients with $\mathrm{HCC}$ who were treated with surgical resection at the University Hospital RWTH Aachen (UH-RWTH) were included in this study. All of these patients had localized tumors without signs of systemic disease. Clinical staging was performed according to the International Union Against Cancer (UICC) criteria. The study was conducted at the UHRWTH in accordance with the requirements of the Institutional Review Board of the RWTH-Aachen University (EK 360/15), the current version of the Declaration of Helsinki, and the good clinical practice guidelines (ICHGCP).

\section{Staging and Surgical Technique}

All patients who were referred for surgical treatment to our institution underwent a detailed clinical work-up as previously described. ${ }^{2,20,21}$ This included the assessment of the number, size, and location of tumor nodules as well as the presence of distant metastases by cross-sectional imaging (gadoliniumbased magnetic resonance imaging (MRI) or contrastmaterial enhanced computed tomography (cmCT)). The patients' perioperative risk was determined based on the American society of anesthesiologists- (ASA) and the Eastern Cooperative Oncology Group (ECOG)-performance status, CT or MRI-based 3D-calculation of the future liver remnant (FLR), as well as the analysis of the quantitative and functional parenchymal liver function as assessed by laboratory parameters and the LiMAx test (Humedics ${ }^{\circledR} \mathrm{GmbH}$, Berlin, Germany). ${ }^{16}$ The decision for hepatectomy as the primary treatment for the individual patient was made by a staff hepatobiliary surgeon and was approved by the institutional interdisciplinary tumor board in all cases. Patients staged BCLC A to BCLC C without any evidence of extrahepatic spread as well as compensated liver function were considered candidates for surgical therapy. Liver resection was carried out in accordance with common clinical standards. An intraoperative ultrasound was performed to visualize the local tumor spread and other suspicious lesions. The decision for either anatomic resections - defined by resection of the related portal vein branch - or non-anatomic atypical wedge resections with an adequate resection margin was based on the 
surgeon's preference. In general, non-anatomic atypical wedge resections were preferred for small, peripherally located, and solitary HCCs that exhibited exophytic growth.

Blood parameters associated with coagulation and transfusion requirement were assessed 1 day prior to surgery in all patients. No FFP or any other blood product was applied prophylactically prior to surgery. The anesthesiologic management comprised restrictive fluid intervention strategy aiming to maintain a low central venous pressure (CVP) during parenchymal dissection. This was further facilitated by reverse Trendelenburg positioning or veno-dilative medication if necessary. During surgery, arterial blood gas analyses were regularly conducted to measure hemoglobin and lactate, while thromboelastography (TEG) was carried out on demand. Parenchymal transection was carried out using the Cavitron Ultrasonic Surgical Aspirator (CUSA $®$, Integra LifeSciences ${ }^{\circledR}$, Plainsboro NJ, USA) with low CVP and intermittent Pringle maneuvers if necessary. Intraoperative blood transfusions were administered based on a restrictive transfusion policy based on an interdisciplinary case-by-case decision between surgeon and anesthesiologist with respect to the amount of blood loss, results of the regularly obtained blood gas analyses and individual co-morbidities. A target hemoglobin concentration of 7-9 $\mathrm{g} / \mathrm{dl}$ was maintained during surgery, and FFPs were administered on a case-by-case decision in cases of coagulopathic bleeding. Platelet transfusions were administered only in cases of pathological TEG results.

The patients were directly transferred to a specialized intensive care unit (ICU) after the procedure. The transfer to a normal postoperative ward was usually carried out on the 1st postoperative day (POD). Patients were later released from the hospital following a final evaluation by the attending surgeon.

All specimens were evaluated for tumor size, histological diagnosis, tumor grading, tumor staging, vessel invasion, resection margin, and presence of cirrhosis by an experienced staff pathologist.

\section{Statistical Analysis}

The primary endpoint of this study was major perioperative inhouse morbidity in HCC patients undergoing surgical resection in curative intent, which was defined as complications rated Clavien-Dindo $\geq 3$ according to the ClavienDindo scale. ${ }^{22}$ The secondary endpoint was the presence of any postoperative complication during hospitalization (Clavien-Dindo $\geq 1$ ). ${ }^{22}$ All complications are reported as inhouse morbidity. Data derived from continuous variables are presented as mean and standard deviation. Associations between perioperative variables and the primary or secondary endpoint were assessed by means of binary logistic regression. Variables showing a $p$ value $<0.1$ in univariate analysis were transferred into a multivariable model and analyzed with multivariable binary logistic regressions using backward elimination. For this purpose, nominal and categorical data were recoded into a scaled dummy variable. Survival curves were generated by the KaplanMeier method under exclusion of perioperative mortality and compared with the log-rank test. Median follow up was accessed with the reverse Kaplan-Meier method. The level of significance was set to $p<0.05$, and $p$ values are given for two-sided testing. Analyses were performed using SPSS Statistics 24 (IBM Corp., Armonk, NY, USA).

\section{Results}

\section{Preoperative, Operative and Postoperative Data}

A total of 136 patients with a mean age of $67 \pm 12$ years and mean body mass index (BMI) of $27 \pm 5 \mathrm{~kg} / \mathrm{m}^{2}$ underwent curative surgery for HCC at our institution from 2011 to 2017. More than half of the patients $(61.1 \%, 83 /$ 136) had a preoperative performance status ASA III or higher assessed by the attending anesthesiologist. The majority of the patients were classified as Child Pugh A (89.7\%, 122/133) with a mean Child Pugh Score (CPS) of $5.3 \pm 0.7$ and a mean model for end-stage liver disease (MELD) of $7 \pm 3$. Milan criteria was fulfilled in 40 patients $(29.4 \%)$. One fifth of the patients $(19.9 \%, 27 / 136)$ underwent laparoscopic liver resection, and mean operative time was $210 \pm 83 \mathrm{~min}$. During surgery, packed red blood cells (RPC) were administered to 33 patients $(27.3 \%)$ and FFP to 51 patients $(42.1 \%)$. The cohort had a mean hospital stay of $14 \pm 13$ days after surgery. Approximately 45\% (62/136) showed no postoperative complications while 40 patients $(29.4 \%)$ experienced major postoperative complications (Clavien-Dindo $\geq 3$ ). The mean postoperative comprehensive complication index (CCI) was $20 \pm 29$. Detailed clinicopathological and perioperative characteristics are outlined in Table 1. Additionally, a concise overview of postoperative complications is presented in Table 2.

\section{Univariate and Multivariable Analysis of Postoperative Morbidity}

A univariate binary logistic regression was carried out for postoperative morbidity (Clavien-Dindo $\geq 1$ ) including all available pre- and intraoperative variables (Table 3 ). Largest tumor diameter $(\mathrm{HR}=2.33 ; p=0.017)$, CPS $(\mathrm{HR}=2.50 ; p=0.047)$, operative time $(\mathrm{HR}=2.08 ; p=$ $0.039)$, FFP transfusion ( $\mathrm{HR}=4.39 ; p=0.001)$, and laparoscopic resection (HR $=0.41 ; p=0.047)$ were associated with postoperative complications (Table 3 ). Variables showing a $p$ value $<0.1$ in univariate analysis were included into a multivariable binary logistic regression model 
Table 1 Clinical and perioperative characteristics

Demographics
Gender, $\mathrm{m} / \mathrm{f}(\%)$
Age $($ years $)$
BMI $\left(\mathrm{kg} / \mathrm{m}^{2}\right)$
Portal vein embolization, $n(\%)$
ASA, $n(\%)$
I
II
III
IV
V
Milan criteria, $n(\%)$
BCLC, $n(\%)$
0
A
B
C
D

Preoperative liver function

MELD Score

Albumin (g/dl)

AST (U/1)

$\operatorname{ALT}(\mathrm{U} / \mathrm{l})$

GGT (U/1)

Total bilirubin $(\mathrm{mg} / \mathrm{dl})$

Platelet count (/nl)

Alkaline Phosphatase (U/l)

Prothrombine time (\%)

INR

Creatinine (mg/dl)

Hemoglobin $(\mathrm{g} / \mathrm{dl})$

Child Pugh, $n(\%)$

$\mathrm{A}$
$\mathrm{B}$
$\mathrm{C}$

Child Pugh score

Operative data

Laparoscopic resection, $\mathrm{n}(\%)$

Conversation rate, $\mathrm{n}(\%)$

Conversion due to bleeding, $\mathrm{n}(\%)$

Operative time (minutes)

Operative procedure, $\mathrm{n}(\%)$

Atypical

Segmentectomy
Bisegmentectomy
Hemihepatectomy
Extended liver resection
other

Additional procedures (RFA, etc.), $n(\%)$

Pringle maneuver, $n(\%)$ mean $\pm \mathrm{SD}$
$96(67.6) / 40(29.7)$
$67 \pm 12$
$27 \pm 5$
$7(6.6)$

0

53 (38.9)

78 (57.4)

$5(3.7)$

0

$40(29.4)$

$3(2.2)$

77 (56.6)

33 (24.3)

19 (14.0)

0

Mean \pm SD

$7 \pm 3$

$39 \pm 7$

$56 \pm 44$

$48 \pm 42$

$172 \pm 173$

$0.6 \pm 0.4$

$239 \pm 112$

$123 \pm 90$

$93 \pm 14$

$1.04 \pm 0.10$

$1.0 \pm 0.8$

$13.1 \pm 1.9$

122 (89.7)

$11(8.1)$

0

$5.3 \pm 0.7$

Mean \pm SD

27 (19.9)

3 (10)

2 (66.7)

$210 \pm 83$

39 (28.7)

13 (16.9)

12 (8.8)

$34(25.0)$

23 (16.9)

5 (3.7)

3 (2.2)

7 (5.2)
Table 1 (continued)

\begin{tabular}{ll}
\hline Duration of Pringle maneuver $(\mathrm{min})^{*}$ & $20(15-25)$ \\
Intraoperative blood transfusion, $n(\%)$ & $33(27.3)$ \\
Intraoperative FFP, $n(\%)$ & $51(42.1)$ \\
Intraoperative platelet transfusion, $n(\%)$ & $3(2.5)$ \\
Pathological examination & mean \pm SD \\
R0 resection, $n(\%)$ & $127(93.4)$ \\
Largest tumor diameter $(\mathrm{mm})$ & $67 \pm 41$ \\
Number of nodules & $1.9 \pm 1.4$ \\
Macrovascular invasion, $n(\%)$ & $32(23.5)$ \\
Tumor stage UICC, $n(\%)$ & \\
I & $48(35.3)$ \\
II & $49(36.0)$ \\
IIIA & $21(15.4)$ \\
IIIB & $8(5.9)$ \\
IIIC & $2(1.5)$ \\
IVA & $3(2.2)$ \\
IVB & $1(0.7)$ \\
Postoperative data & mean \pm SD \\
Intensive care stay, days & $2 \pm 9$ \\
Hospitalization, days & $14 \pm 13$ \\
Postoperative complications, $n(\%)$ & \\
No complications & $62(45.6)$ \\
Clavien-Dindo I & $19(14.0)$ \\
Clavien-Dindo II & $15(11.0)$ \\
Clavien-Dindo IIIa & $15(11.0)$ \\
Clavien-Dindo IIIb & $8(5.9)$ \\
Clavien-Dindo IVa & $7(5.1)$ \\
Clavien-Dindo IVb & $1(0.7)$ \\
Clavien-Dindo V & $9(6.6)$ \\
CCI & $20 \pm 29$ \\
Postoperative liver failure & $2(1.5)$ \\
Postoperative blood transfusion & $22(18.2)$ \\
Postoperative FFP & $9(7.4)$ \\
Postoperative platelet transfusion & $5(4.1)$ \\
\hline &
\end{tabular}

Data presented as mean and standard deviation if not noted otherwise. *Median and interquartile range. ${ }^{\text {* }}$ Postoperative liver failure was assessed by the 50-50-criteria. $A L T$, alanine aminotransferase; $A S A$, American society of anesthesiologists classification; $A S T$, aspartate aminotransferase; $B C L C$, Barcelona clinical liver cancer staging system; $B M I$, body mass index; $C C I$, comprehensive complication index; $F F P$, fresh frozen plasma; $G G T$, gamma glutamyltransferase; INR, international normalized ratio; $M E L D$, model of end stage liver disease; $M W A$, microwave ablation; UICC, Union for International Cancer Control

which determined FFP $(\mathrm{HR}=4.39 ; p=0.001)$ as the single significant predictor of postoperative morbidity (Table 4). For major postoperative morbidity (Clavien-Dindo $\geq 3$ ), the univariable analysis showed significant associations of the largest tumor diameter ( $\mathrm{HR}=2.55 ; p=0.023)$, CPS $(\mathrm{HR}=2.60 ; p=0.030)$, operative time $(\mathrm{HR}=3.84 ; p=$ $0.003)$, blood transfusions $(\mathrm{HR}=2.51 ; p=0.035)$, FFP transfusion ( $\mathrm{HR}=6.52 ; p=0.001)$, and major postoperative complications (Table 3 ). Variables showing a $p$ value $<0.1$ in univariate analysis were again included in the corresponding multivariable binary logistic regression model which determined CPS (HR $=3.23 ; p=0.040)$, operative time $(\mathrm{HR}=5.63 ; \mathrm{p}=0.003)$ and FFP $(\mathrm{HR}=5.62 ; \mathrm{p}=$ 0.001 ) as independent predictors of major postoperative morbidity (Table 5). 
Table 2 Detailed overview of postoperative complications

\begin{tabular}{|c|c|}
\hline Postoperative complication & $n(\%)$ \\
\hline \multicolumn{2}{|l|}{ Sub-cohort with intraoperative FFP } \\
\hline Bile leakage & $9(23.7)$ \\
\hline Pneumonia & $5(13.2)$ \\
\hline Pleural effusion & $4(10.5)$ \\
\hline Ascites & $2(5.3)$ \\
\hline Postoperative hemorrhage & $2(5.3)$ \\
\hline Surgical site infection & $2(5.3)$ \\
\hline Unspecific infection & $2(5.3)$ \\
\hline Pulmonary embolism & $2(5.3)$ \\
\hline Acute renal failure & $2(5.3)$ \\
\hline Cardiac arrhythmia & $2(5.3)$ \\
\hline Urinary tract infection & $1(2.6)$ \\
\hline Liver insufficiency & $1(2.6)$ \\
\hline Portal vein thrombosis & $1(2.6)$ \\
\hline Delirium & $1(2.6)$ \\
\hline Prolonged postoperative nausea & $1(2.6)$ \\
\hline Prolonged postoperative pain & $1(2.6)$ \\
\hline \multicolumn{2}{|c|}{ Sub-cohort without intraoperative FFP } \\
\hline Bile leakage & $4(14.8)$ \\
\hline Urinary tract infection & $3(11.1)$ \\
\hline Ascites & $3(11.1)$ \\
\hline Electrolyte disorders & $3(11.1)$ \\
\hline Septic shock & $2(7.4)$ \\
\hline Postoperative hemorrhage & $2(7.4)$ \\
\hline Surgical site infection & $1(3.7)$ \\
\hline Pleural effusion & $1(3.7)$ \\
\hline Unspecific infection & $1(3.7)$ \\
\hline Pulmonary embolism & $1(3.7)$ \\
\hline Acute renal failure & $1(3.7)$ \\
\hline Pancreatitis & $1(3.7)$ \\
\hline Paralytic ileus & $1(3.7)$ \\
\hline Pneumothorax & $1(3.7)$ \\
\hline Allergic reaction & $1(3.7)$ \\
\hline Intraabdominal fluid collection & $1(3.7)$ \\
\hline
\end{tabular}

The leading postoperative complication was assessed in every patient who experienced postoperative complications. FFP, fresh frozen plasma

A similar analysis regarding postoperative and major postoperative morbidity was carried out for the subcohort of patients who received intraoperative FFP $(n=$ 51). Here, no statistical significance was found except for operative time in association to major postoperative morbidity $(\mathrm{HR}=7.33 ; p=0.006$; Supplementary Table S1). Also, we conducted a group comparison regarding operative characteristics of patients with and without intraoperative FFP transfusion. In this analysis, no difference was observed regarding the particular surgical procedure $(p=$ $0.305)$, laparoscopic approach $(p=0.135)$, and the utilization of Pringle maneuver ( $p=0.111)$ except for a longer operative time in patients receiving FFP (230 \pm 86 min vs. $196 \pm 82 \mathrm{~min} ; p=0.027$; Supplementary Table S2). No difference was observed in perioperative mortality $(7.8 \%, 4 / 51$ vs. $4.3 \%, 3 / 70 ; p=0.408)$ and postoperative liver failure $(3.9 \%(2 / 50)$ vs. $0 / 70 ; p=0.095)$ between patients with and without intraoperative FFP transfusion.

We further assessed the relationship between intraoperatively transfused FFP and preoperative liver function parameters. Here, no significant association was observed between FFP transfusion and preoperative serum albumin $(p=0.671)$, bilirubin $(p=0.767)$, CPS $(p=0.055)$, International normalized Ratio (INR, $p=0.517$ ), MELD $(p=0.120)$, platelet count $(p=0.301)$, and prothrombin time ( $p=0.392)$ (Table 6).

No statistical associations were observed between intraoperatively and postoperatively applied blood transfusions $(p=0.160)$, FFP $(p=0.193)$, and platelet transfusions ( $p=$ $0.869)$.

\section{Survival Analysis}

With a median follow-up of 3.5 years, the OS in our cohort was 3.5 years (95\% confidence interval $(\mathrm{CI}), 1.6-5.4$ years). No association was observed between oncological outcome and postoperative morbidity ( $p=0.345 \log$ rank), major postoperative morbidity ( $p=0.611 \log$ rank), and intraoperative FFP administration ( $p=0.110$ log rank) (Fig. 1).

\section{Discussion}

The management and prevention of perioperative morbidity have become an important goal in modern-era HCC surgery. Here, we aimed to evaluate the association of various clinicopathological parameters with perioperative outcomes in HCC-patients undergoing surgical resection in curative intent. Our multivariable model identified intraoperative FFP transfusion as an independent prognostic marker for overall morbidity and intraoperative FFP transfusion, CPS, and operative time as independent predictors for major morbidity.

The adverse effects of blood products on surgical and oncological outcomes in HCC have been demonstrated earlier. ${ }^{24-26}$ It is generally assumed that blood transfusions adversely affect long-term outcomes, especially in patients with early-stage HCCs. ${ }^{27-29}$ While the effects of blood transfusions in liver surgery have been investigated before, the exact significance of FFP transfusion in clinical outcomes in HCC patients undergoing partial hepatectomy in curative intent remains to be determined. ${ }^{30}$ Historically, Japanese centers advocate a relatively liberal usage of 
Table 3 Univariable analysis of perioperative morbidity

\begin{tabular}{|c|c|c|c|c|c|c|c|}
\hline & \multirow[t]{2}{*}{$n$} & \multicolumn{3}{|c|}{ Major morbidity (Clavien-Dindo $\geq 3$ ) } & \multicolumn{3}{|c|}{ Morbidity (Clavien-Dindo $\geq 1$ ) } \\
\hline & & Hazard ratio & $95 \% \mathrm{CI}$ & $P$ value & Hazard ratio & $95 \% \mathrm{CI}$ & $P$ value \\
\hline Sex & & & & 0.467 & & & 0.773 \\
\hline Male & 96 & & & & & & \\
\hline Female & 40 & & & & & & \\
\hline Age, years & & & & 0.540 & & & 0.517 \\
\hline$\leq 65$ & 53 & & & & & & \\
\hline$>65$ & 83 & & & & & & \\
\hline BMI, $\mathrm{kg} / \mathrm{m}^{2}$ & & & & 0.733 & & & 0.893 \\
\hline$\leq 25$ & 54 & & & & & & \\
\hline$>25$ & 82 & & & & & & \\
\hline ASA & & & & 0.812 & & & 0.865 \\
\hline $\mathrm{I} / \mathrm{II}$ & 62 & & & & & & \\
\hline III/IV & 74 & & & & & & \\
\hline Milan criteria & & & & 0.063 & & & 0.172 \\
\hline Yes & 40 & & & & & & \\
\hline No & 95 & & & & & & \\
\hline BCLC Staging & & & & 0.587 & & & 0.445 \\
\hline 0 & 3 & & & & & & \\
\hline A & 77 & & & & & & \\
\hline $\mathrm{B}$ & 33 & & & & & & \\
\hline $\mathrm{C}$ & 19 & & & & & & \\
\hline $\mathrm{D}$ & 0 & & & & & & \\
\hline Largest tumor diameter, $\mathrm{mm}$ & & & & 0.023 & & & 0.017 \\
\hline$\leq 50$ & 59 & 1 & & & 1 & & \\
\hline$>50$ & 76 & 2.55 & $1.14-5.69$ & & 2.33 & $1.16-4.67$ & \\
\hline Number of nodules & & & & 0.427 & & & 0.582 \\
\hline Single & 84 & & & & & & \\
\hline Multilocular & 49 & & & & & & \\
\hline Macrovascular invasion & & & & 0.443 & & & 0.584 \\
\hline No & 99 & & & & & & \\
\hline Yes & 32 & & & & & & \\
\hline MELD & & & & 0.130 & & & 0.112 \\
\hline$\leq 8$ & 61 & & & & & & \\
\hline$>8$ & 73 & & & & & & \\
\hline Child Pugh score & & & & 0.030 & & & 0.047 \\
\hline$\leq 5$ & 108 & 1 & & & 1 & & \\
\hline$>5$ & 28 & 2.60 & $1.10-6.15$ & & 2.50 & $1.01-6.16$ & \\
\hline Albumin, g/l & & & & 0.579 & & & 0.537 \\
\hline$\leq 40$ & 46 & & & & & & \\
\hline$>40$ & 54 & & & & & & \\
\hline AST, U/1 & & & & 0.303 & & & 0.811 \\
\hline$\leq 40$ & 55 & & & & & & \\
\hline$>40$ & 66 & & & & & & \\
\hline ALT, U/1 & & & & 0.598 & & & 0.765 \\
\hline$\leq 40$ & 57 & & & & & & \\
\hline$>40$ & 49 & & & & & & \\
\hline GGT, U/1 & & & & 0.713 & & & 0.486 \\
\hline$\leq 100$ & 58 & & & & & & \\
\hline$>100$ & 62 & & & & & & \\
\hline Bilirubin, $\mathrm{mg} / \mathrm{dl}$ & & & & 0.826 & & & 0.521 \\
\hline$\leq 1$ & 61 & & & & & & \\
\hline$>1$ & 73 & & & & & & \\
\hline Alkaline phosphatase, $\mathrm{U} / \mathrm{l}$ & & & & 0.508 & & & 0.228 \\
\hline$\leq 100$ & 60 & & & & & & \\
\hline$>100$ & 59 & & & & & & \\
\hline
\end{tabular}


Table 3 (continued)

\begin{tabular}{|c|c|c|c|c|c|c|c|}
\hline & \multirow[t]{2}{*}{$n$} & \multicolumn{3}{|c|}{ Major morbidity (Clavien-Dindo $\geq 3$ ) } & \multicolumn{3}{|c|}{ Morbidity (Clavien-Dindo $\geq 1$ ) } \\
\hline & & Hazard ratio & $95 \% \mathrm{CI}$ & $P$ value & Hazard ratio & $95 \% \mathrm{CI}$ & $P$ value \\
\hline Platelet count, $1 / \mathrm{nl}$ & & & & 0.152 & & & 0.616 \\
\hline$\leq 200$ & 54 & & & & & & \\
\hline$>200$ & 80 & & & & & & \\
\hline Prothrombin time, $\%$ & & & & 0.240 & & & 0.613 \\
\hline$\leq 100$ & 105 & & & & & & \\
\hline$>100$ & 29 & & & & & & \\
\hline INR & & & & 0.304 & & & 0.626 \\
\hline$\leq 1$ & 47 & & & & & & \\
\hline$>1 ;<1.2$ & 75 & & & & & & \\
\hline$\geq 1.2$ & 12 & & & & & & \\
\hline Creatinine, $\mathrm{mg} / \mathrm{dl}$ & & & & 0.952 & & & 0.343 \\
\hline$<1$ & 61 & & & & & & \\
\hline$\geq 1$ & 74 & & & & & & \\
\hline Hemoglobin, g/dl & & & & 0.189 & & & 0.283 \\
\hline$\leq 12$ & 42 & & & & & & \\
\hline$>12$ & 92 & & & & & & \\
\hline Operative time, $\min$ & & & & 0.003 & & & 0.039 \\
\hline$\leq 180$ & 55 & 1 & & & 1 & & \\
\hline$>180$ & 81 & 3.84 & $1.60-9.18$ & & 2.08 & $1.04-4.18$ & \\
\hline Intraoperative blood transfusion & & & & 0.035 & & & 0.104 \\
\hline No & 88 & 1 & & & & & \\
\hline Yes & 33 & 2.51 & $1.07-5.87$ & & & & \\
\hline Intraoperative FFP & & & & 0.001 & & & 0.001 \\
\hline No & 70 & 1 & & & 1 & & \\
\hline Yes & 51 & 6.52 & $2.68-15.86$ & & 4.39 & $1.99-9.67$ & \\
\hline Intraoperative platelet transfusion & & & & 0.841 & & & 0.666 \\
\hline No & 118 & & & & & & \\
\hline Yes & 3 & & & & & & \\
\hline Laparoscopic resection & & & & 0.481 & & & 0.047 \\
\hline No & 109 & & & & 1 & & \\
\hline Yes & 27 & & & & 0.41 & $0.17-0.99$ & \\
\hline Type of surgery & & & & 0.412 & & & 0.614 \\
\hline Atypical & 39 & & & & & & \\
\hline Segmentectomy & 23 & & & & & & \\
\hline Bisegmentectomy & 12 & & & & & & \\
\hline Hemihepatectomy & 34 & & & & & & \\
\hline Extended liver resection & 23 & & & & & & \\
\hline other & 5 & & & & & & \\
\hline Pringle maneuver & & & & .417 & & & .630 \\
\hline No & 127 & & & & & & \\
\hline Yes & 7 & & & & & & \\
\hline
\end{tabular}

Various parameters are associated with major and general postoperative morbidity. Hazard ratios are shown for statistically significant variables. $A L T$, alanine aminotransferase; $A S A$, American society of anesthesiologists classification; $A S T$, aspartate aminotransferase; $B C L C$, Barcelona clinical liver cancer staging system; $B M I$, body mass index; $F F P$, fresh frozen plasma; $G G T$, gamma glutamyltransferase; INR, international normalized ratio; $M E L D$, model of end stage liver disease; UICC, Union for International Cancer Control. *Mean

FFPs compared with European and North-American centers, which propagate more restrictive administration of FFPs in this setting. ${ }^{31}$ Tomimaru et al. compared longterm outcome of 297 patients with FFP transfusion on demand with a historical cohort of 204 patients which routinely received FFP postoperatively and found no difference in oncologic outcome. ${ }^{32}$ A further Japanese report also concluded that the administration of FFP-in contrast to RPC-does not influence survival of patients undergoing partial hepatectomy for $\mathrm{HCC}^{33}$ Although FFPs have already shown an adverse effect on oncologic outcome in other malignancies (pancreatic cancer, 
Table 4 Multivariable binary logistic regression of any perioperative morbidity

\begin{tabular}{llll}
\hline Variable & \multicolumn{2}{l}{ Morbidity (Clavien-Dindo $\geq 1)$} \\
\cline { 2 - 4 } & Hazard Ratio & $95 \%$ CI & P value \\
\hline Largest tumor diameter, mm & & & 0.228 \\
$\leq 50$ & 1 & & \\
$>50$ & 1.63 & $0.74-3.60$ & \\
Child Pugh score & & & 0.198 \\
$\leq 5$ & 1 & & \\
$>5$ & 1.96 & $0.70-5.45$ & \\
Operative time, min & & & 0.149 \\
$\leq 180$ & 1 & & \\
$>180$ & 1.82 & $0.81-4.10$ & \\
FFP & & & 0.001 \\
No & 1 & $1.99-9.67$ & \\
Yes & 4.39 & & 0.213 \\
Laparoscopic resection & & & \\
No & 1 & $0.21-1.41$ & \\
Yes & .549 & & \\
\hline
\end{tabular}

All variables showing statistical significance in univariate binary logistic regression were included in a multivariable logistic regression. Hazard ratios are shown for statistically significant variables. $F F P$, fresh frozen plasma

Table 5 Multivariable binary logistic regression of major perioperative morbidity

\begin{tabular}{|c|c|c|c|}
\hline \multirow[t]{2}{*}{ Variable } & \multicolumn{3}{|c|}{ Morbidity (Clavien Dindo $\geq 3$ ) } \\
\hline & Hazard Ratio & $95 \% \mathrm{CI}$ & $P$ value \\
\hline Largest tumor diameter, $\mathrm{mm}$ & & & 0.809 \\
\hline$\leq 50$ & 1 & & \\
\hline$>50$ & 1.20 & $0.27-5.38$ & \\
\hline Child Pugh score & & & 0.040 \\
\hline$\leq 5$ & 1 & & \\
\hline$>5$ & 3.23 & $1.06-9.87$ & \\
\hline Operative time, $\min$ & & & 0.003 \\
\hline$\leq 180$ & 1 & & \\
\hline$>180$ & 5.63 & $1.82-17.41$ & \\
\hline Blood transfusions & & & 0.395 \\
\hline No & 1 & & \\
\hline Yes & .608 & $0.19-1.91$ & \\
\hline FFP & & & 0.001 \\
\hline No & 1 & & \\
\hline Yes & 5.62 & $2.19-14.40$ & \\
\hline Milan criteria & & & 0.635 \\
\hline Yes & 1 & & \\
\hline No & 1.51 & $.28-8.19$ & \\
\hline
\end{tabular}

All variables showing statistical significance in univariate binary logistic regression were included in a multivariable logistic regression. Hazard ratios are shown for statistically significant variables. FFP, fresh frozen plasma
Table 6 Intraoperatively applied FFP units in relation to liver function parameters

\begin{tabular}{|c|c|c|c|}
\hline \multirow[t]{2}{*}{ Variable } & \multicolumn{3}{|c|}{ FFP units } \\
\hline & Mean & Standard deviation & $P$ value \\
\hline Albumin, g/l & & & 0.671 \\
\hline$\leq 40$ & 1.98 & 2.68 & \\
\hline$>40$ & 1.77 & 2.55 & \\
\hline Bilirubin, mg/dl & & & 0.767 \\
\hline$\leq 1$ & 1.89 & 2.58 & \\
\hline$>1$ & 2.14 & 2.91 & \\
\hline Child Pugh Score & & & 0.055 \\
\hline$\leq 5$ & 1.72 & 2.51 & \\
\hline$>5$ & 2.80 & 2.94 & \\
\hline INR & & & 0.517 \\
\hline$\leq 1$ & 2.13 & 2.79 & \\
\hline$>1$ & 1.78 & 2.50 & \\
\hline MELD & & & 0.120 \\
\hline$\leq 8$ & 1.80 & 2.62 & \\
\hline$>8$ & 2.56 & 2.53 & \\
\hline Platelet count, $1 / \mathrm{nl}$ & & & 0.301 \\
\hline$\leq 200$ & 2.26 & 2.88 & \\
\hline$>200$ & 1.67 & 2.37 & \\
\hline Prothrombin time, $\%$ & & & 0.392 \\
\hline$\leq 100$ & 1.80 & 2.54 & \\
\hline$>100$ & 2.28 & 2.83 & \\
\hline
\end{tabular}

No statistical difference in intraoperative FFP transfusion between patients grouped by liver function parameters was observed. FFP, fresh frozen plasma; INR, international normalized ratio

colorectal liver metastases), clinical evidence suggesting a similar effect in HCC is lacking. ${ }^{34,35}$

Shiba et al. assessed the perioperative complications in 99 patients undergoing hepatectomy for HCC. In this cohort, pulmonary complications were associated with the cumulative count of perioperatively transfused FFPs in multivariable analysis. Unlike our study, Shiba et al. evaluated the role of cumulatively transfused FFPs in both the intra- and postoperative course. ${ }^{36}$ Therefore, the present study is the first to report on the adverse effects of intraoperatively transfused FFPs in terms of perioperative outcome in patients with HCC. The requirement of postoperative FFPs administration is commonly attributed to the lack of clotting factors in association with a postoperative liver dysfunction. Moreover, liver failure per se is associated with an increased rate of bacterial infections, and the onset of hepatopulmonary syndrome might also influence the rate of pulmonary complications. ${ }^{37}$ Therefore, postoperative FFP transfusion is mostly required in cases with significant postoperative liver dysfunction/failure which might explain the observed predisposition to postoperative complications 


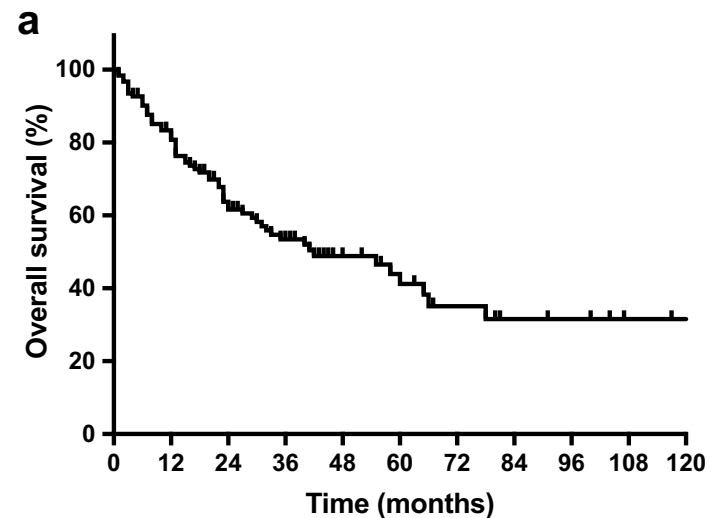

C

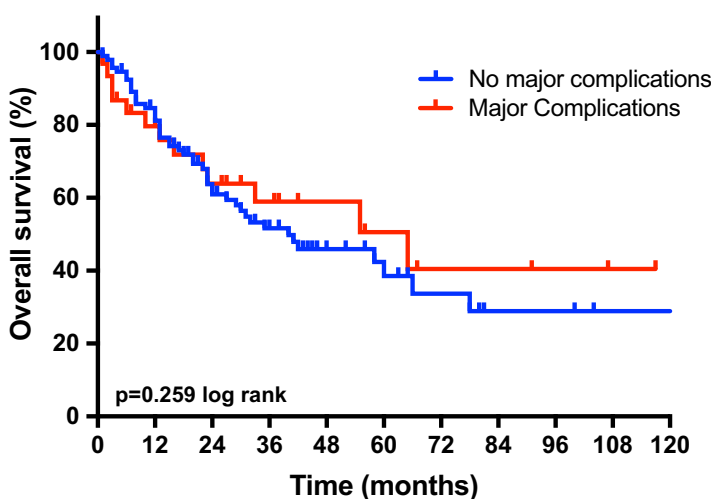

Fig. 1 Oncological survival in hepatocellular carcinoma a Overall survival in hepatocellular carcinoma. The median OS of the cohort was 3.5 years (95\% CI: 1.6-5.4). b Overall survival in hepatocellular carcinoma stratified by postoperative complications. The Kaplan-Meier analysis with respect to postoperative complications showed a median OS of 4.8 years $(95 \% \mathrm{CI}, 2.2-7.5)$ in patients without postoperative complications compared to 3.3 years (95\% CI, 1.4-5.3) in patients with postoperative complications ( $p=0.892 \mathrm{log}$ rank). $\mathbf{c}$ Overall survival in hepatocellular carcinoma stratified by major postoperative complications. The Kaplan-Meier analysis with respect to major postoperative complications

in the above-mentioned study of Shiba et al. This fact makes it difficult to interpret a causal relationship between postoperative FFP administration and perioperative outcomes.

Clot formation can be facilitated by the maintenance of core body temperature $>35^{\circ} \mathrm{C}, \mathrm{pH}>7.2$ and plasma calcium levels $>1 \mathrm{mmol} / \mathrm{L}{ }^{38}$ From a surgical point of view, transfusion requirements can also be reduced by surgical technique and the adherence to low CVP by restrictive transfusion and volume administration policy during parenchymal dissection. ${ }^{39}$ It is reasonable to assume that the need of intraoperative transfusion may also be associated with the underlying chronic liver disease. In our study, the preoperative assessment of the liver function was not associated with postoperative morbidity. Also, we did not find a relationship between preoperative liver function and intraoperatively transfused FFPs, highlighting the importance of an optimized intraoperative blood management (Table 6).
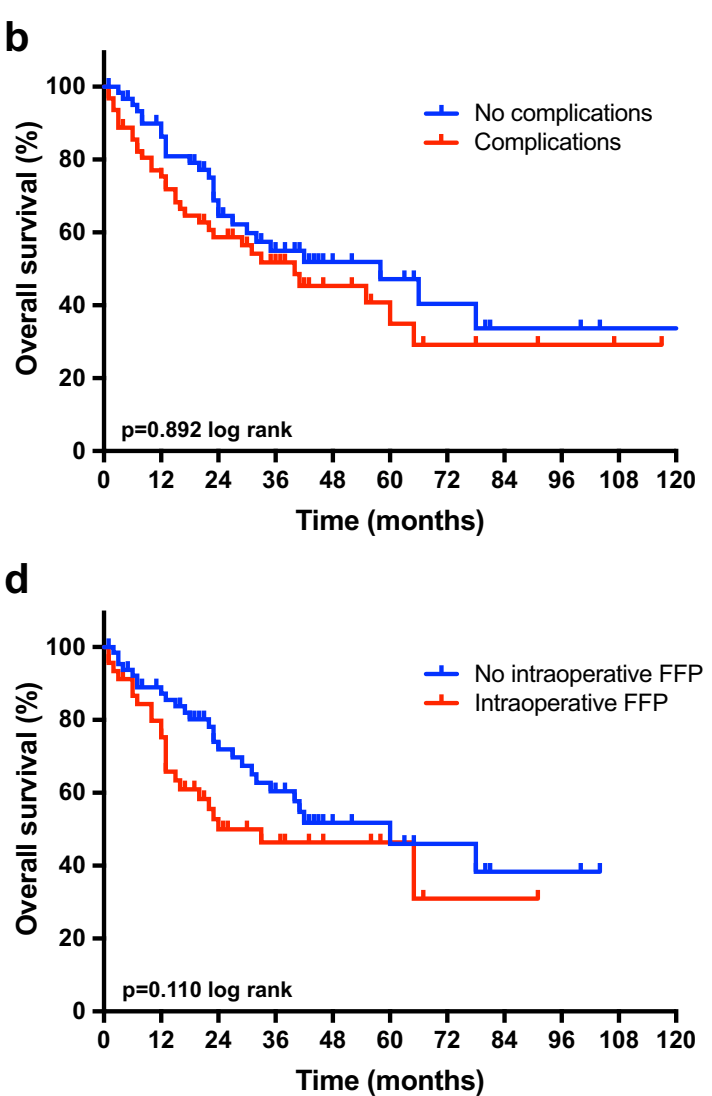

(Clavien-Dindo $\geq 3$ ) showed a median OS of 3.3 years (95\% CI, 1.4-5.2) in patients without major postoperative complications compared to 5.4 years $(95 \%$ CI, 1.8-9.0) in patients with major postoperative complications ( $p=0.259 \log$ rank). d Overall survival in hepatocellular carcinoma stratified by intraoperative FFP. The Kaplan-Meier analysis with respect to intraoperative administration of FFP showed a median OS of 5.0 years $(95 \% \mathrm{CI}, 2.2-7.8)$ in patients who have not received intraoperative FFP compared to 2.0 years $(95 \% \mathrm{CI}, 0-4.3)$ in patients who have received intraoperative $\operatorname{FFP}(p=0.110 \log$ rank). CI, confidence interval; FFP, fresh frozen plasma; OS, overall survival

The association of FFP transfusion with perioperative morbidity in partial hepatectomy for HCC might be explained with the effect of transfusion-related immunomodulation. As such, Sarani et al. found a correlation between transfusion of FFP and pulmonary or blood stream infections in critically ill surgical patients. ${ }^{40}$ Some investigators speculated that soluble proteins in FFP may cause similar immunosuppressive effects as seen with RBC transfusions. ${ }^{40}$ Such proteins may include human leukocyte antigen and fibrinogen/fibrin degradation products or disrupted white blood cell products. These components can be found in FFPs, even after leukoreduction and may alter the immune response. ${ }^{41}$ Discussed mechanisms include diminished antigen processing by macrophages, upregulation of both $\mathrm{T}$ suppressor and regulatory cells and humoral immunosuppressive mediators, impaired natural killer cell activity, and 
production of anti-idiotypic antibodies. ${ }^{41}$ However, there is no definitive or satisfactory explanation for transfusionrelated immunomodulation and many pathophysiological aspects of this phenomenon remain to be determined. Interestingly, the transfusion-related immunomodulation and the discussed pathomechanisms are also linked to increased rates of cancer recurrence. ${ }^{41}$ Thus, future studies should explore the association between FFP and oncologic outcome as it has already been demonstrated for other malignancies. ${ }^{34,35}$

Like any other perioperative outcome study, our analysis has certain inherent limitations. All patients included in this study were treated at a single institution reflecting our local clinical approach and the study is based on retrospective data which were not obtained in the setting of a controlled prospective clinical trial. As such, our transfusion strategy did not follow a strict study protocol. Further, our sample size is relatively small compared to some other studies especially from Asian cohorts. Therefore, further subset analyses of patients who received intraoperative FFPs were not possible. However, due to the lower incidence of HCC in Europe, European studies with significantly larger sample sizes are mostly multi-center analyses with different clinical approaches to this complex disease, while all patients of our cohort were treated according to the same clinical standards. Also, in eastern and western specialized centers, HCC patients present with different epidemiologic and clinical characteristics, including tumor biology and clinical behavior, necessitating slightly different therapeutic approaches. Thus, results and conclusions from Asian cohorts might not be applicable to Western patients. ${ }^{42,43}$

Notwithstanding the aforementioned limitations, we have identified intraoperative administration of FFPs as an important predictor of perioperative morbidity in patients undergoing liver resections for HCC. A more restrictive policy in terms of intraoperative FFP transfusions by optimizing surgical and anesthesiologic conditions should be a key goal in partial hepatectomy for HCC. Larger, prospective clinical trials are needed to confirm and validate our findings in HCC and other hepatic malignancies.

\section{Acknowledgments Open Access funding provided by Projekt DEAL.}

Authorship The study was designed by JB, ZC, UPN and GL. Data was collected by ZC, IL, PS, NTG and AB. Data was analyzed by JB, TL, PB and TFU. Data was interpreted by JB, CT, SAL, UPN and GL. The initial manuscript was drafted by JB, UPN and GL and critically revised by ZC, IL, CT, TL, PS, NTG, AB, PB, TFU and SAL. All authors have read and approved the final version of the manuscript. All authors do also agree to be accountable for all aspects of the work.

Funding information This research project is supported by the STARTProgram (\#136/17) of the Faculty of Medicine, RWTH Aachen and funded by the Excellence Initiative of the German federal and state governments (G:(DE-82) ZUK2-SF-OPSF486). The funders were not involved in study design, data collection, data analysis, manuscript preparation or decision to publish.

\section{Compliance with Ethical Standards}

Conflict of Interest The authors have no conflict of interest to disclose.

Open Access This article is licensed under a Creative Commons Attribution 4.0 International License, which permits use, sharing, adaptation, distribution and reproduction in any medium or format, as long as you give appropriate credit to the original author(s) and the source, provide a link to the Creative Commons licence, and indicate if changes were made. The images or other third party material in this article are included in the article's Creative Commons licence, unless indicated otherwise in a credit line to the material. If material is not included in the article's Creative Commons licence and your intended use is not permitted by statutory regulation or exceeds the permitted use, you will need to obtain permission directly from the copyright holder. To view a copy of this licence, visit http://creativecommons.org/licenses/by/4.0/.

\section{References}

1. Siegel R, Naishadham D, Jemal A. Cancer statistics for Hispanics/ Latinos, 2012. CA: a cancer journal for clinicians. 2012;62(5):28398. doi:https://doi.org/10.3322/caac.21153.

2. Lurje G, Bednarsch J, Czigany Z, Amygdalos I, Meister F, Schoning $\mathrm{W}$ et al. Prognostic factors of disease-free and overall survival in patients with hepatocellular carcinoma undergoing partial hepatectomy in curative intent. Langenbeck's archives of surgery / Deutsche Gesellschaft fur Chirurgie. 2018;403(7):851-61. doi:https://doi.org/10.1007/s00423-018-1715-9.

3. Llovet JM, Burroughs A, Bruix J. Hepatocellular carcinoma. Lancet. 2003;362(9399):1907-17. doi:https://doi.org/10.1016/ S0140-6736(03)14964-1.

4. Lurje G, Lesurtel M, Clavien PA. Multimodal treatment strategies in patients undergoing surgery for hepatocellular carcinoma. Dig Dis. 2013;31(1):112-7. doi:https://doi.org/10.1159/000347205.

5. Czigany Z, Schoning W, Ulmer TF, Bednarsch J, Amygdalos I, Cramer $\mathrm{T}$ et al. Hypothermic oxygenated machine perfusion (HOPE) for orthotopic liver transplantation of human liver allografts from extended criteria donors (ECD) in donation after brain death (DBD): a prospective multicentre randomised controlled trial (HOPE ECD-DBD). BMJ Open. 2017;7(10):e017558. doi:https:// doi.org/10.1136/bmjopen-2017-017558.

6. Teh SH, Nagorney DM, Stevens SR, Offord KP, Therneau TM, Plevak DJ et al. Risk factors for mortality after surgery in patients with cirrhosis. Gastroenterology. 2007;132(4):1261-9. doi:https:// doi.org/10.1053/j.gastro.2007.01.040.

7. Teh SH, Christein J, Donohue J, Que F, Kendrick M, Farnell M et al. Hepatic resection of hepatocellular carcinoma in patients with cirrhosis: Model of End-Stage Liver Disease (MELD) score predicts perioperative mortality. Journal of gastrointestinal surgery : official journal of the Society for Surgery of the Alimentary Tract. 2005;9(9):1207-15; discussion 15. doi:https://doi.org/10.1016/j. gassur.2005.09.008.

8. Maithel SK, Kneuertz PJ, Kooby DA, Scoggins CR, Weber SM, Martin RC, 2nd et al. Importance of low preoperative platelet count in selecting patients for resection of hepatocellular carcinoma: a multi-institutional analysis. Journal of the American College of Surgeons. 2011;212(4):638-48; discussion 48-50. doi:https://doi. org/10.1016/j.jamcollsurg.2011.01.004. 
9. Stockmann M, Lock JF, Riecke B, Heyne K, Martus P, Fricke M et al. Prediction of postoperative outcome after hepatectomy with a new bedside test for maximal liver function capacity. Annals of surgery. 2009;250(1):119-25. doi:https://doi.org/10.1097/SLA. 0b013e3181ad85b5.

10. Lurje I, Czigany Z, Bednarsch J, Roderburg C, Isfort P, Neumann UP et al. Treatment Strategies for Hepatocellular Carcinoma (-) a Multidisciplinary Approach. Int J Mol Sci. 2019;20(6). doi:https:// doi.org/10.3390/ijms20061465.

11. Nguyen KT, Marsh JW, Tsung A, Steel JJ, Gamblin TC, Geller DA. Comparative benefits of laparoscopic vs open hepatic resection: a critical appraisal. Archives of surgery. 2011;146(3):348-56. doi:https://doi.org/10.1001/archsurg.2010.248.

12. de Graaf W, van Lienden KP, Dinant S, Roelofs JJ, Busch OR, Gouma DJ et al. Assessment of future remnant liver function using hepatobiliary scintigraphy in patients undergoing major liver resection. Journal of gastrointestinal surgery : official journal of the Society for Surgery of the Alimentary Tract. 2010;14(2):369-78. doi:https://doi.org/10.1007/s11605-009-1085-2.

13. Imamura H, Sano K, Sugawara Y, Kokudo N, Makuuchi M. Assessment of hepatic reserve for indication of hepatic resection: decision tree incorporating indocyanine green test. Journal of hepato-biliary-pancreatic surgery. 2005;12(1):16-22. doi:https:// doi.org/10.1007/s00534-004-0965-9.

14. Imamura H, Seyama Y, Kokudo N, Maema A, Sugawara Y, Sano $\mathrm{K}$ et al. One thousand fifty-six hepatectomies without mortality in 8 years. Archives of surgery. 2003;138(11):1198-206; discussion 206. doi:https://doi.org/10.1001/archsurg.138.11.1198.

15. Jara M, Reese T, Malinowski M, Valle E, Seehofer D, Puhl G et al. Reductions in post-hepatectomy liver failure and related mortality after implementation of the LiMAx algorithm in preoperative workup: a single-centre analysis of 1170 hepatectomies of one or more segments. HPB : the official journal of the International Hepato Pancreato Biliary Association. 2015;17(7):651-8. doi:https://doi. org/10.1111/hpb.12424.

16. Takayama T. Surgical treatment for hepatocellular carcinoma. Japanese journal of clinical oncology. 2011;41(4):447-54. doi: https://doi.org/10.1093/jjco/hyr016.

17. Hanazaki K, Kajikawa S, Shimozawa N, Mihara M, Shimada K, Hiraguri $\mathrm{M}$ et al. Survival and recurrence after hepatic resection of 386 consecutive patients with hepatocellular carcinoma. Journal of the American College of Surgeons. 2000;191(4):381-8.

18. Mizuguchi T, Kawamoto M, Meguro M, Nakamura Y, Ota S, Hui TT et al. Prognosis and predictors of surgical complications in hepatocellular carcinoma patients with or without cirrhosis after hepatectomy. World journal of surgery. 2013;37(6):1379-87. doi: https://doi.org/10.1007/s00268-013-1989-6.

19. Doussot A, Lim C, Lahat E, Salloum C, Osseis M, Gavara CG et al. Complications after Hepatectomy for Hepatocellular Carcinoma Independently Shorten Survival: A Western, Single-Center Audit. Annals of surgical oncology. 2017;24(6):1569-78. doi:https://doi. org/10.1245/s10434-016-5746-6.

20. Bednarsch J, Czigany Z, Lurje I, Tacke F, Strnad P, Ulmer TF et al. Left- versus right-sided hepatectomy with hilar en-bloc resection in perihilar cholangiocarcinoma. HPB : the official journal of the International Hepato Pancreato Biliary Association. 2019. doi: https://doi.org/10.1016/j.hpb.2019.07.003.

21. Lurje G, Bednarsch J, Czigany Z, Lurje I, Schlebusch IK, Boecker J et al. The prognostic role of lymphovascular invasion and lymph node metastasis in perihilar and intrahepatic cholangiocarcinoma. Eur J Surg Oncol. 2019. doi:https://doi.org/10.1016/j.ejso.2019.04. 019.

22. Dindo D, Demartines N, Clavien PA. Classification of surgical complications: a new proposal with evaluation in a cohort of 6336 patients and results of a survey. Annals of surgery. 2004;240(2):205-13.
23. Balzan S, Belghiti J, Farges O, Ogata S, Sauvanet A, Delefosse D et al. The "50-50 criteria" on postoperative day 5: an accurate predictor of liver failure and death after hepatectomy. Annals of surgery. 2005;242(6):824-8, discussion 8-9.

24. Katz SC, Shia J, Liau KH, Gonen M, Ruo L, Jarnagin WR et al. Operative blood loss independently predicts recurrence and survival after resection of hepatocellular carcinoma. Annals of surgery. 2009;249(4):617-23. doi:https://doi.org/10.1097/SLA. 0b013e31819ed22f.

25. Shiba H, Ishida Y, Wakiyama S, Iida T, Matsumoto M, Sakamoto T et al. Negative impact of blood transfusion on recurrence and prognosis of hepatocellular carcinoma after hepatic resection. Journal of gastrointestinal surgery : official journal of the Society for Surgery of the Alimentary Tract. 2009;13(9):1636-42. doi:https://doi.org/ 10.1007/s11605-009-0963-y.

26. Harada N, Shirabe K, Maeda T, Kayashima H, Ishida T, Maehara Y. Blood transfusion is associated with recurrence of hepatocellular carcinoma after hepatectomy in Child-Pugh class A patients. World journal of surgery. 2015;39(4):1044-51. doi:https://doi.org/10. 1007/s00268-014-2891-6.

27. Yang T, Lu JH, Lau WY, Zhang TY, Zhang H, Shen YN et al. Perioperative blood transfusion does not influence recurrence-free and overall survivals after curative resection for hepatocellular carcinoma: A Propensity Score Matching Analysis. Journal of hepatology. 2016;64(3):583-93. doi:https://doi.org/10.1016/j.jhep. 2015.10.012.

28. Kuroda S, Tashiro H, Kobayashi T, Oshita A, Amano H, Ohdan H. No impact of perioperative blood transfusion on recurrence of hepatocellular carcinoma after hepatectomy. World journal of surgery. 2012;36(3):651-8. doi:https://doi.org/10.1007/s00268-0121425-3.

29. de Boer MT, Molenaar IQ, Porte RJ. Impact of blood loss on outcome after liver resection. Digestive surgery. 2007;24(4):259-64. doi:https://doi.org/10.1159/000103656.

30. Bennett S, Baker L, Shorr R, Martel G, Fergusson D. The impact of perioperative red blood cell transfusions in patients undergoing liver resection: a systematic review protocol. Syst Rev. 2016;5:38. doi: https://doi.org/10.1186/s13643-016-0217-5.

31. Takagi T, Kajiro K, Yazawa Y, Takada Y, Takeda T, Teratani M et al. A Study on appropriate usage of fresh frozen plasma and albumin for patients in internal medicine. Journal of the Japan Society of Blood Transfusion. 2004;50(6).

32. Tomimaru Y, Wada H, Marubashi S, Kobayashi S, Eguchi H, Takeda $\mathrm{Y}$ et al. Fresh frozen plasma transfusion does not affect outcomes following hepatic resection for hepatocellular carcinoma. World journal of gastroenterology : WJG. 2010;16(44):5603-10.

33. Kaibori M, Saito T, Matsui K, Yamaoka M, Kamiyama Y. Impact of fresh frozen plasma on hepatectomy for hepatocellular carcinoma. Anticancer research. 2008;28(3B):1749-55.

34. Shiba H, Ishida Y, Haruki K, Furukawa K, Fujiwara Y, Iwase R et al. Negative impact of fresh-frozen plasma transfusion on prognosis after hepatic resection for liver metastases from colorectal cancer. Anticancer research. 2013;33(6):2723-8.

35. Qiu L, Wang DR, Zhang XY, Gao S, Li XX, Sun GP et al. Impact of perioperative blood transfusion on immune function and prognosis in colorectal cancer patients. Transfusion and apheresis science : official journal of the World Apheresis Association : official journal of the European Society for Haemapheresis. 2016;54(2): 235-41. doi:https://doi.org/10.1016/j.transci.2015.07.004.

36. Shiba H, Ishii Y, Ishida $\mathrm{Y}$, Wakiyama $\mathrm{S}$, Sakamoto T, Ito R et al. Assessment of blood-products use as predictor of pulmonary complications and surgical-site infection after hepatectomy for hepatocellular carcinoma. Journal of hepato-biliary-pancreatic surgery. 2009;16(1):69-74. doi:https://doi.org/10.1007/s00534-008-0006-1.

37. Fernandez J, Acevedo J, Wiest R, Gustot T, Amoros A, Deulofeu C et al. Bacterial and fungal infections in acute-on-chronic liver 
failure: prevalence, characteristics and impact on prognosis. Gut. 2017. doi:https://doi.org/10.1136/gutjnl-2017-314240.

38. Meybohm P, Zacharowski K, Weber CF. Point-of-care coagulation management in intensive care medicine. Critical care. 2013;17(2): 218. doi:https://doi.org/10.1186/cc12527.

39. Weeder PD, Porte RJ, Lisman T. Hemostasis in liver disease: implications of new concepts for perioperative management. Transfus Med Rev. 2014;28(3):107-13. doi:https://doi.org/10.1016/j.tmrv. 2014.03.002.

40. Sarani B, Dunkman WJ, Dean L, Sonnad S, Rohrbach JI, Gracias VH. Transfusion of fresh frozen plasma in critically ill surgical patients is associated with an increased risk of infection. Critical care medicine. 2008;36(4):1114-8. doi:https://doi.org/10.1097/ CCM.0b013e318168f89d.
41. Refaai MA, Blumberg N. Transfusion immunomodulation from a clinical perspective: an update. Expert Rev Hematol. 2013;6(6): 653-63. doi:https://doi.org/10.1586/17474086.2013.850026.

42. Vibert E, Ishizawa T. Hepatocellular carcinoma: Western and Eastern surgeons' points of view. Journal of visceral surgery. 2012;149(5):e302-6. doi:https://doi.org/10.1016/j.jviscsurg.2012. 05.001 .

43. Herman P, Coelho FF. Laparoscopic resection for hepatocellular carcinoma: eastern and western experiences. Chin J Cancer Res. 2014;26(3):234-6. doi:https://doi.org/10.3978/j.issn.1000-9604. 2014.06.19.

Publisher's Note Springer Nature remains neutral with regard to jurisdictional claims in published maps and institutional affiliations. 